Additional Perspectives articles for Influenza: The Cutting Edge book collection are available at http://perspectivesinmedicine.cshlp.org/cgi/collection/influenza_the_cutting_edge.

\title{
Human Influenza Epidemiology
}

\author{
Sukhyun Ryu ${ }^{1}$ and Benjamin J. Cowling ${ }^{2}$ \\ ${ }^{1}$ Department of Preventive Medicine, Konyang University College of Medicine, Daejeon 35365, South Korea \\ ${ }^{2}$ WHO Collaborating Centre for Infectious Disease Epidemiology and Control, School of Public Health, Li Ka \\ Shing Faculty of Medicine, The University of Hong Kong, Hong Kong Special Administrative Region, China \\ Correspondence: bcowling@hku.hk
}

Influenza virus infections are common in people of all ages. Epidemics occur in the winter months in temperate locations and at varying times of the year in subtropical and tropical locations. Most influenza virus infections cause mild and self-limiting disease, and around one-half of all infections occur with a fever. Only a small minority of infections lead to serious disease requiring hospitalization. During epidemics, the rates of influenza virus infections are typically highest in school-age children. The clinical severity of infections tends to increase at the extremes of age and with the presence of underlying medical conditions, and impact of epidemics is greatest in these groups. Vaccination is the most effective measure to prevent infections, and in recent years influenza vaccines have become the most frequently used vaccines in the world. Nonpharmaceutical public health measures can also be effective in reducing transmission, allowing suppression or mitigation of influenza epidemics and pandemics.

$\mathrm{H}$ undreds of millions of influenza virus infections occur around the world each year. Although immunity against reinfection with the same strain can last for many years, rapid antigenic change in circulating strains allows influenza viruses to escape the population immunity that builds up after an epidemic (Saad-Roy et al. 2019; Lam et al. 2020). Occasionally, influenza pandemics occur when a novel strain emerges from animals and is able to spread among humans (Saunders-Hastings and Krewski 2016). Whereas most infections are associated with mild and self-limiting disease, older individuals and those with underlying medical conditions can be more vulnerable to more serious disease and influenza epidemics tend to have the great- est impact in these groups. Influenza can also cause serious disease in neonates and infants. Here, we review the incidence and severity profile of influenza virus infections in populations, and the public health impact of those epidemics. We then discuss the measures that can be used to control influenza epidemics and pandemics.

\section{INFLUENZA TRANSMISSION}

Transmission of an infectious disease occurs when the causative pathogen moves from an infected host to cause an infection in a new host. The rate at which transmission occurs will define the speed and impact of an epidemic, and public health measures typically aim to slow

Editors: Gabriele Neumann and Yoshihiro Kawaoka

Additional Perspectives on Influenza: The Cutting Edge available at www.perspectivesinmedicine.org

Copyright (C) 2020 Cold Spring Harbor Laboratory Press; all rights reserved

Advanced Online Article. Cite this article as Cold Spring Harb Perspect Med doi: 10.1101/cshperspect.a038356 
down or stop transmission from occurring. At the beginning of an epidemic, in a completely susceptible population with no public health measures in place, transmissibility can be measured by the basic reproductive number, often written as " $R_{0}$." This quantity is formally defined as the mean number of secondary infectious cases generated from a typical primary infectious case in a completely susceptible population. One review study reported that the median basic reproduction number for the 1918 pandemic was 1.80 (interquartile range [IQR]: 1.47-2.27), 1.65 (IQR: 1.53-1.70) for the 1957 pandemic, 1.80 (IQR: $1.56-1.85$ ) for the 1968 pandemic, 1.46 (IQR: $1.30-1.70$ ) for the 2009 pandemic, and 1.28 (IQR: 1.19-1.37) for seasonal influenza (Biggerstaff et al. 2014). One implication of this is that between one-third and one-half of all transmission events must be prevented by public health measures to bring the reproductive number below the critical threshold of 1 and stop an epidemic.

As infections spread, leading to a buildup of immunity in the population, transmissibility will gradually decline. Changes over time in transmissibility can be reflected by a metric known as the effective reproduction number at time $t\left(R_{t}\right)$, which is the number of secondary infections generated by a typical infection with illness onset at calendar time $t$. As an epidemic proceeds, $R_{t}$ will tend to decline, and will drop below the critical threshold of 1 at around the epidemic peak. Monitoring $R_{t}$ can also give information on the impact of public health measures on transmission (Ali et al. 2018; Cowling et al. 2020; Ryu et al. 2020a).

Understanding the ways in which influenza transmission occurs is important to determine which types of control measures would be most appropriate to recommend. For example, if influenza often spreads through contaminated surfaces, hand hygiene would be a particularly important and efficacious intervention. Most influenza transmission is thought to occur during close contact by droplets or aerosols that travel through the air from one person to another, without involving hands or fomites. However, the size of droplets or aerosols responsible for transmission remains a topic of considerable debate (Killingley and Nguyen-Van-Tam 2013; Tellier et al. 2019).

\section{SEASONALITY IN DIFFERENT CLIMATE ZONES}

In temperate locations, influenza epidemics tend to occur in the winter months. Influenza seasonality is weaker in subtropical and tropical locations, and influenza can occur at various times of the year or even have year-round circulation (Hirve et al. 2016; Dave and Lee 2019). There are a number of proposed mechanisms that affect this seasonality. One hypothesis is that seasonality is affected by the variation of host immunity against infectious agents-for example, because of seasonal changes in melatonin or vitamin D levels (Dowell 2001). Another potential mechanism is behavioral change, in which populations spend more time indoors during the winter, whereas schools tend to have holidays in the summer. A third possible mechanism of seasonality is the change in survival of influenza virus under different environmental conditions such as humidity (Shaman and Kohn 2009; Marr et al. 2019).

\section{INCIDENCE OF INFECTIONS}

Measures of influenza incidence include the number or rate of infections that have occurred, are occurring, or are predicted to occur in a population. In some cases, measures of incidence will focus on influenza virus infections with certain clinical characteristics, such as ambulatory consultations, or hospitalizations associated with influenza. Because many influenza virus infections are mild and do not require medical attention, it is challenging to measure the incidence of all infections in a population. Laboratory diagnosis can be costly and may be unnecessary for management of most influenza virus infections. A typical approach to influenza surveillance has been to measure the proportion of outpatients with influenza-like illness (ILI) over time (Fleming and Elliot 2008). Surveillance of inpatients is another routine approach, in which the proportion of inpatients with severe acute respiratory infections is monitored 
over time. In most influenza epidemics, the incidence of infections is higher in children than adults. One recent study estimated that $\sim 3 \%-11 \%$ of individuals in the United States suffer symptomatic influenza each year, with an average incidence of $8.7 \%$ in children and $5.1 \%$ in adults (Tokars et al. 2018).

In the 2009 influenza pandemic, laboratory diagnostics were more widely used particularly among inpatients, although it can be difficult to interpret incidence rates of laboratory-confirmed cases if testing practices vary over time. Virologic surveillance could provide accurate information on relative changes in incidence of infections over time as long as health-care-seeking behaviors and testing practices are stable over time. Combining surveillance of medically attended ILI with virologic data on the proportion of ILI associated with influenza has been used as a correlate of the incidence of infections in some studies (Goldstein et al. 2011; Wong et al. 2013b; Yang et al. 2015).

In addition to surveillance of medically attended influenza, infections and illness can also be measured by epidemiological studies in the general community (Monto 1994). Other elec- tronic data sources could be used to track incidence: For example, calls to a telenursing system could inform rates of milder illnesses, one could monitor Internet searches relating to influenza that could correlate with rates of infections, or if resources are available one could arrange for repeated telephone surveys to assess rates of ILIs over time (Payne et al. 2005; Ginsberg et al. 2009; Spreco et al. 2017).

The immune response to an influenza virus infection can include production of antibodies specific to that strain, and serologic surveillance of the prevalence of antibody in a population over time can provide direct information on the cumulative incidence of infections. An example of this is shown in Figure $1 \mathrm{~A}$, based on a serological study done in Hong Kong in 2009 (Wu et al. 2010b). However, some technical issues must be addressed, including uncertainty over the proportion of infected individuals who develop antibody at or above a certain titer (particularly among those with subclinical infections), the delays in appearance of antibody, waning in antibody titers over the longer term, potential cross-reactions due to infections with other strains, and con-
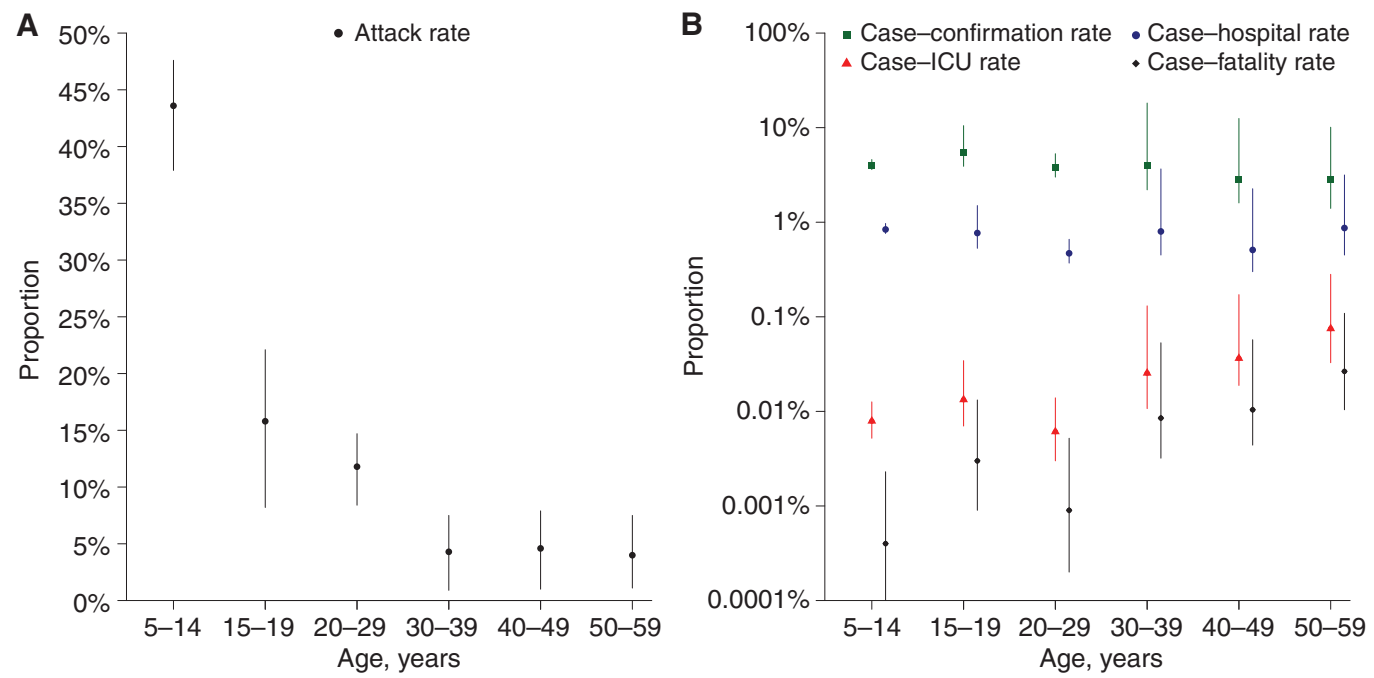

Figure 1. Age-specific infection attack rate and clinical severity profile of $2009 \mathrm{H} 1 \mathrm{~N} 1 \mathrm{pdm} 09$ infections in Hong Kong (Wu et al. 2010b). (A) Estimates of the risk of infection in each age group based on a large serologic study (Wu et al. 2010b). (B) Age-specific estimates of the clinical severity profile of H1N1pdm09 in Hong Kong based on the estimated attack rates and the numbers of severe outcomes recorded in the territory. 
fusion of changes in antibody titer following vaccination versus infection (Chen et al. 2018; Khurana et al. 2019).

Incidence can also be measured in closed settings such as schools and households, and outbreak investigations or transmission studies can provide valuable data on infections and illnesses. For example, prospective follow-up of individuals exposed to infection can provide data on the proportion of virologically confirmed infections that lead to certain clinical signs or symptoms or increases in antibody titers, facilitating interpretation of surveillance data from the broader population (Tsang et al. 2016).

One of the key determinants of the incidence of an influenza strain is its transmissibility, or its propensity to spread in a population. Transmissibility is affected by the degree of infectiousness of those infected, the susceptibility of those in contact with infected individuals, and the degree of contact between infectious and susceptible individuals. Transmissibility is not a biological constant and can be modified by population behaviors and public health measures. Tracking transmissibility over time allows public health authorities to determine the strength of measures needed to bring an epidemic under control.

\section{SERIOUSNESS OR SEVERITY PROFILE OF INFECTIONS}

"Seriousness," or severity, describes the pathogenicity or the capacity of an influenza strain to cause disease. One of the most common measures of seriousness is the case fatality risk (Nishiura 2010), often referred to as the case fatality rate, although it is not a rate (Kelly and Cowling 2013). The numerator is the estimated number of deaths, the denominator is the estimated number of cases, and it is important to clarify the specific measures used for each of these. In the early stages of an epidemic, some infections may not have resolved, so that the number of deaths divided by the number of cases could underestimate the case fatality risk if some of the current cases would later die (Jewell et al. 2007). It is possible to include only deaths of laboratory-confirmed cases in the numerator, or broaden to all estimated influenza-associated deaths (Wong et al. 2013b). It is possible to restrict the denominator to laboratory-confirmed cases only (which depends on health-care-seeking behavior and the availability of laboratory testing) (Nishiura 2010), broaden to all symptomatic individuals with a certain definition of "symptomatic," or even broaden further to all estimated infections including subclinical infections (Wu et al. 2010b, 2011; Wong et al. 2013b). Variability in definitions for both the numerator and denominator can lead to problems in comparing severity estimates in different settings. In 2009, a study in Hong Kong was able to estimate the seriousness profile of H1N1pdm09 infections by age (Fig. 1B), by comparing estimates of the incidence of infection from serological data (Fig. 1A) with estimates of the numbers of hospitalizations and deaths (Wu et al. 2010b). In Figure 1B, there is a clear increase with age in the risk of serious outcomes on a per-infection basis: For example, the mortality rate was $<1$ per 100,000 infections in children, whereas it was approaching 1 per 1000 infections in adults 50-59 years of age and likely would be higher than that in older adults.

Seriousness is not limited to measures of the risk of mortality, but could more generally refer to the risk of having a more serious health outcome: for example, the risk of illness conditional on infection, the risk of hospitalization conditional on illness, the risk of death conditional on hospitalization, etc. Alternatively, a simple definition of the seriousness of a particular influenza strain could be proposed as the average cost associated with treating an infection with that strain either in terms of direct costs or including indirect costs as well. However, single measures of severity may not fully capture the profile of an influenza strain as the risk of severe disease increases with age and with the presence of other chronic health conditions (Van Kerkhove et al. 2011).

Seriousness could also be reflected at the mild end of the spectrum by the fraction of infections that are asymptomatic, the fraction of infections that cause a febrile disease, or a disease that requires medical attention. However, 
these fractions are challenging to estimate. Estimates of the asymptomatic fraction of infections have varied between $10 \%$ and $90 \%$, depending on the approaches used and the precise definition of "asymptomatic" (Leung et al. 2015). In community studies with intense monitoring of exposed persons, around one-half of infections are associated with a fever (Lau et al. 2012; Ip et al. 2016).

Prospective assessment of the likely impact of an influenza epidemic or pandemic can help to determine the types of public health measures that might be justified to reduce transmission. In March 2017, the World Health Organization (WHO) published the Pandemic Influenza Severity Assessment approach to improve and standardize severity assessment and to enable a real-time assessment to guide response (World Health Organization 2017b). The components in this assessment include transmissibility of influenza, the seriousness of disease, and the potential impact on the society as a whole. The seriousness profile of infections is typically the most difficult parameter to evaluate in real time (Wong et al. 2013a).

\section{THE PUBLIC HEALTH IMPACT OF INFLUENZA VIRUS INFECTIONS}

Influenza epidemics and pandemics can have considerable morbidity and mortality impact. The influenza H1N1 pandemic in 1918/1919 had a particularly high impact (Pappas et al. 2008). It had three separate waves during 1918-1919 and caused 50-80 million deaths (Taubenberger and Morens 2006). Two other influenza pandemics occurred in the twentieth century with lower impact (Table 1). In April
2009, H1N1pdm09 was identified in Mexico and the United States, and infections rapidly spread worldwide. In its first year of circulation, it has been estimated that H1N1pdm09 caused between 123,000 and 203,000 deaths (Simonsen et al. 2013).

The impact of influenza in a population can be measured by the number of illnesses, outpatient visits, hospitalizations, and/or deaths associated with infections in that population. In addition to counts of the number of health events, measures of impact can be broadened to encompass economic considerations (Klepser 2014), mental health and well-being (Coughlin 2012), and indirect effects on other diseases (Noymer 2011; Mak et al. 2012). Economic costs can reflect not only the directly attributable costs of infections, such as inpatient and outpatient care, and workplace absenteeism, but also intangible costs due to changes in population behaviors.

Seasonal influenza tends to be associated with a substantial morbidity and mortality impact in older adults and those with underlying medical conditions. Because previous influenza pandemics have been associated with a greater mortality impact in younger individuals, either because of increases in the incidence of infection or severity or both, metrics such as years of life lost have been used to give a more complete picture of the mortality impact than the number of deaths (Charu et al. 2011; Zhou et al. 2013).

One approach to estimating the mortality impact of influenza is by examining the "excess" deaths that occur during influenza epidemics compared to outside of epidemic periods (Farr 1847). Influenza-associated mortality varies from year to year and tends to be higher in pan-

Table 1. Influenza pandemics in the twentieth and twenty-first centuries

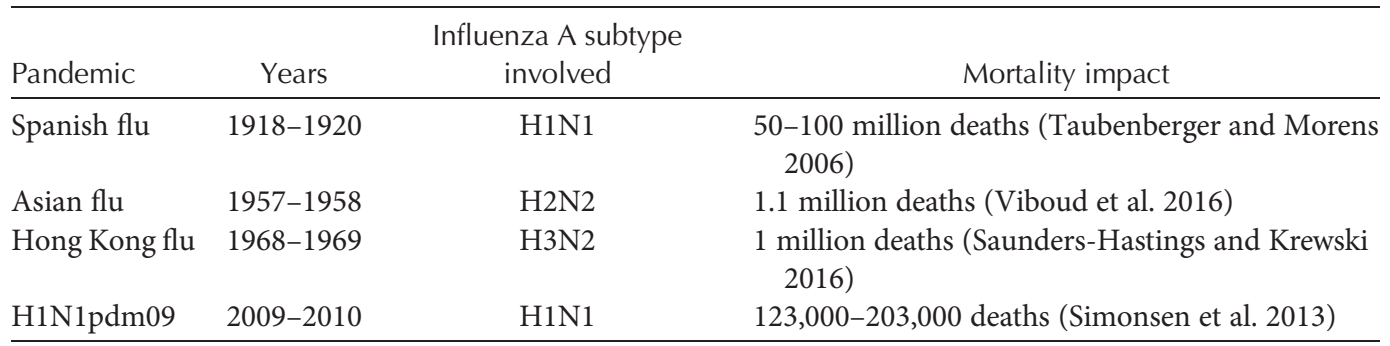


demics than in seasonal epidemics (Nicoll et al. 2012). First, the factors were associated with real differences in influenza-associated death. This includes the characteristics of the virus such as virulence and transmissibility, population structure such as access to health care, the prevalence of commodities, and level of population immunity. Second, the factors were associated with the methodology of case identification. This includes different types of mortality surveillance, differences in laboratory testing, accessibility of test- and case-reporting systems, and different physician's awareness of influenza tests.

Influenza-attributable mortality has been widely estimated by measuring the excess mortality during influenza epidemics compared with baseline threshold, which are usually acquired from statistical and modeling methods (Li et al. 2018). The Serfling least-squares cyclical regression model is one of the most widely used methods, relying on a sinusoidal baseline curve fitted to historical mortality data (Serfling 1963). This model does not require virological data and provides a simple approach to estimate excess deaths, although it is not suitable for locations with weak influenza seasonality. Regression models, which include virological surveillance data, are often now preferred for estimating influenza virus-type and subtypespecific mortality and can avoid overestimation of deaths caused by cold weather or other respiratory infections.

Another approach is counting the number of influenza deaths. However, there are a number of limitations to this approach. First, mortality associated with influenza could be underestimated because infected individuals may not be identified by medical institutions or infected individuals identified at the medical institution but not confirmed by laboratory testing. In addition, influenza virus infections may cause secondary bacterial pneumonia (Davis et al. 2012) or may aggravate underlying illness such as cardiovascular diseases. Influenza virus infections are thought to lead to increases in cardiovascular events, although these are rarely recorded as influenza-associated events because influenza is not suspected in the etiology of these events and virus shedding may already have ceased by the time of the event (Warren-Gash et al. 2009, 2018; Morris et al. 2017).

A recent study estimated that the global mean annual influenza-associated respiratory excess mortality rate ranged from 0.1 to 6.4 per 100,000 individuals for the individuals younger than 65 years, $2.9-44.0$ per 100,000 individuals in those between 65 and 74 years, and 17.9-224.5 per 100,000 individuals for those older than 75 years of age (Iuliano et al. 2018). The highest mortality was in sub-Saharan Africa (2.8-16.5 per 100,000 population) and Southeast Asia (3.5-9.2 per 100,000 population) (Iuliano et al. 2018). These estimates indicate a greater annual burden than the previous report in 2017 (WHO estimated that 290,000 to 650,000 annual global deaths were associated with seasonal influenza) (World Health Organization 2017a).

\section{OPTIONS FOR INFLUENZA CONTROL}

\section{Vaccines}

Influenza vaccines are the best available intervention to reduce the public health impact of influenza epidemics and pandemics. Influenza vaccines have been used since the 1940s with vaccines mostly produced by inactivation of egg-grown virus followed by purification (Sautto et al. 2018). Vaccines have moderate effectiveness in preventing laboratory-confirmed influenza with effectiveness varying from year to year for a variety of reasons (Belongia et al. 2016). Nevertheless, even with moderate effectiveness, influenza vaccines prevent a considerable number of illnesses, hospitalizations, and deaths each year (Chung et al. 2020). There is currently a major international research effort to develop improved influenza vaccines and ultimately "universal" influenza vaccines, which provide broad and long-lasting protection against a wide range of strains (Erbelding et al. 2018; Paules et al. 2018).

Annual monitoring of influenza vaccination effectiveness has become an important public health activity in many high-income locations in recent years. To achieve this, a more efficient study design is required than randomized con- 
trolled trials, which require substantial resources, or classical observational studies that can suffer various biases including confounding (Jackson et al. 2006). Over the last few years, the "test-negative design" has been increasingly used for monitoring vaccine effectiveness (Sullivan et al. 2014). This study design is a variation of the case-control study, in which patients are enrolled if they visited an outpatient clinic (or were hospitalized, in some studies) with symptoms of influenza and were tested for the presence of influenza virus. Vaccination effectiveness can be measured by calculating the odds of vaccination coverage between the enrolled patients testing positive and negative for influenza (Jackson and Nelson 2013).

\section{Antivirals}

There are a number of antivirals with proven efficacy for the treatment of influenza virus infections. Some antivirals can also be used for prophylaxis. In general, most randomized trials of antivirals have been conducted against mild and self-limiting disease, and there is relatively less evidence for their efficacy in treating severe influenza (Kelly and Cowling 2015). However, in practice, antivirals are most useful for treating severe influenza and are less commonly used in outpatient settings. Some countries have created stockpiles of antiviral drugs to be used in the event of an influenza pandemic; although these stockpiles are typically not large enough to treat every mild case, they do provide reassurance that there will be a sufficient supply of effective drugs for severe cases.

\section{Nonpharmaceutical Interventions}

Whereas vaccines are the best preventive measure, nonpharmaceutical interventions (NPIs) also have an important role in mitigating influenza epidemics and pandemics in the community. NPIs can also be described as public health measures. In the early phase of influenza epidemics and pandemics, NPIs are often the most accessible interventions because of the limited supply of antivirals and a delay in the availability of specific vaccines. These measures are intended to reduce individuals' risk of infection, delay the epidemic peak, reduce the "height" of the epidemic peak, and spread cases over a long time period. Achieving any of these objectives would contribute to reducing the overall impact of the epidemic or pandemic (Fig. 2). Notably, health systems often struggle in larger seasonal influenza epidemics, with surges in hospitalizations that can exceed the available bed capacity. In a severe pandemic, spreading out infections over a longer period of time would be vital to preserve hospital capacity and capacity in intensive care facilities in

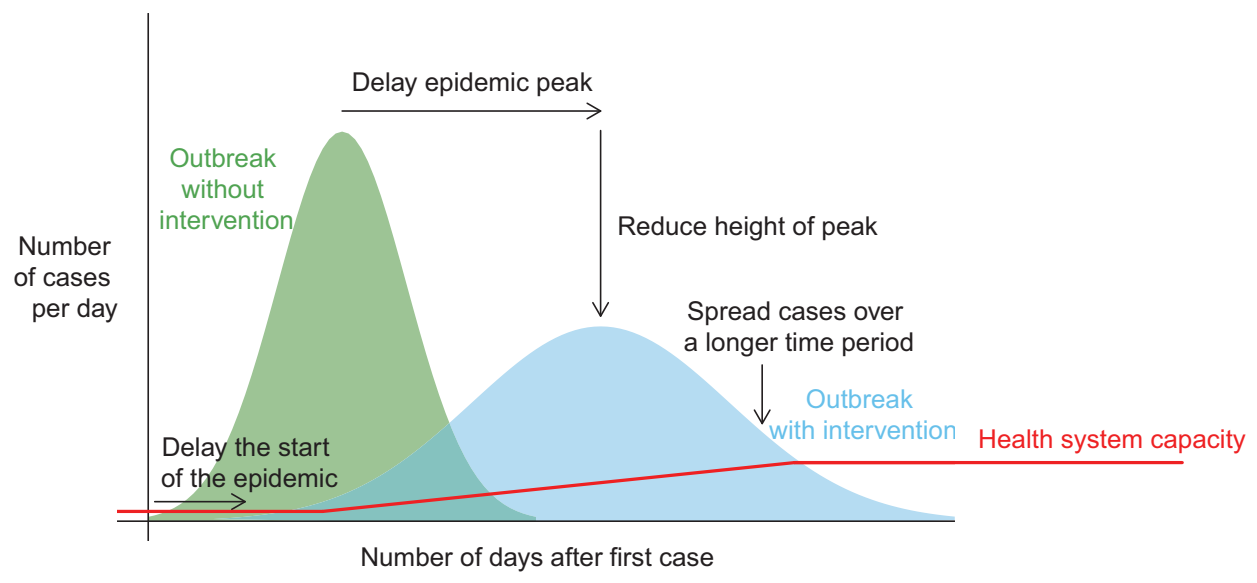

Figure 2. The intended impact of nonpharmaceutical interventions, indicating how "flattening the curve" could allow a greater fraction of patients to be effectively managed by the available health system capacity and could buy time for capacity to be increased. 
particular. Figure 2 indicates the problem with limited health system capacity, which can surpass $100 \%$ capacity even in average influenza seasons. "Flattening the curve" with NPIs could allow time for capacity to be increased. During the COVID-19 pandemic in 2020, NPIs bought time for construction of field hospitals to provide extra capacity for patient isolation and treatment.

Personal protective measures such as hand hygiene and respiratory etiquette are often recommended in influenza epidemics and pandemics, although there is limited evidence for their effectiveness (Xiao et al. 2020). A randomized controlled trial of hand hygiene in 60 elementary school children in Egypt found that hand hygiene significantly reduced laboratoryconfirmed influenza cases by $>50 \%$ (Talaat et al. 2011). However, other randomized trials did not identify significant effects of hand hygiene (Xiao et al. 2020).

Face masks have been one of the most controversial public health measures in the COVID19 pandemic of 2020. There is mechanistic evidence supporting the efficacy of face masks in filtering influenza virus if worn by infected persons (Milton et al. 2013; Leung et al. 2020). However, there is less evidence for their effectiveness in reducing transmission in the community, and a meta-analysis of randomized trials did not identify significant effects (Xiao et al. 2020). It remains plausible, from all available evidence, that widespread use of face masks could have a small-to-moderate effect in reducing influenza transmission in the community.

Isolation of infected persons can be an effective way to reduce transmission (Fong et al. 2020). One of the most important public health messages in an influenza epidemic can be for ill persons to stay at home. The duration of home isolation could be until a fever subsides or for the first few days of illness. One complication would be the access to sick pay, particularly for selfemployed or contract workers, because otherwise these individuals may need to continue to work while ill.

Quarantine is defined as the restriction of movement of exposed persons who are often identified by contact tracing. This has proven to be an important measure in the COVID-19 pandemic. A simulation study estimated that the combination of contact tracing, quarantine, isolation, and antivirals could reduce infections by 40\% (Wu et al. 2006); however, another simulation study estimated that it would be difficult to control influenza with $90 \%$ contact tracing and quarantine because such a high proportion of influenza virus infections are asymptomatic or present with mild symptoms (Fraser et al. 2004).

Social distancing measures are measures intended to reduce the frequency and duration of interactions among people in the community. School-age children usually have lower levels of immunity than adults because they have had fewer prior influenza virus infections, and there are more opportunities for transmission to occur in crowded classrooms compared to other community settings. It is well recognized that school-age children play a crucial role in influenza transmission in the community. To limit the spread of influenza, school closures are one of the most effective social distancing measures available (Fong et al. 2020). The impact of school closures against influenza transmission has been discussed in modeling and epidemiological studies (Cauchemez et al. 2008; Wu et al. 2010a). Transmission has sometimes rebounded when schools are reopened, indirectly indicating that the closures had been suppressing influenza transmission (Jackson et al. 2013; Ryu et al. 2020a). School closure can also reduce transmission in other age groups because of the changes in social mixing. Other important social distancing measures include prevention of mass gatherings, public health measures in workplaces such as work-at-home policies, and measures in institutions such as prisons and residential care homes.

In the early stages of the 2009 influenza pandemic, there was an effort by many countries to delay the importation of infections. Travel-related NPIs include screening travelers for signs or symptoms of infection, travel restrictions, and border closures. Screening arriving travelers can be done with thermal scanners and health declaration forms. Ecological studies estimated that entry screening delayed the importation of 
H1N1pdm09 infections in 2009 by 7-12 days (Cowling et al. 2010), and delayed local epidemics by 4 days (Yu et al. 2012); however, the detection rate of the influenza cases by entry screening is low (Hale et al. 2012; Sakaguchi et al. 2012) and requires considerable public health resources. Therefore, it may not always be justifiable. International travel restriction is the prevention of movement between particular countries. Travel restrictions or reductions are more controversial because of the economic consequences, and simulation studies predicted that very substantial travel restrictions of up to 99\% might be necessary to delay local epidemics by a few weeks (Cooper et al. 2006; Ferguson et al. 2006). Border closure involves the complete limitation of movement of the person into and out of a particular country. Border closure in 11 South Pacific Island jurisdictions delayed the arrival of 1918/1919 pandemic influenza by 3-30 months (McLeod et al. 2008). However, border closures are not likely to be practical or feasible in most parts of the world (World Health Organization 2007; Ryu et al. 2020b).

\section{CONCLUSIONS}

Influenza epidemics cause considerable morbidity and mortality each year, and influenza pandemics threaten to cause as much impact as coronavirus disease 2019. We need an improved understanding of the modes of influenza transmission to design better control measures. Influenza vaccines are increasingly used to mitigate the impact of epidemics but require annual administration and have moderate effectiveness that could be improved by the development of universal influenza vaccines.

\section{ACKNOWLEDGMENTS}

The authors thank Julie Au for technical assistance.

This article has been made freely available online courtesy of TAUNS Laboratories.

\section{REFERENCES}

Ali ST, Cowling BJ, Lau EHY, Fang VJ, Leung GM. 2018. Mitigation of influenza B epidemic with school closures, Hong Kong, 2018. Emerg Infect Dis 24: 2071-2073. doi:10 .3201/eid2411.180612

Belongia EA, Simpson MD, King JP, Sundaram ME, Kelley NS, Osterholm MT, McLean HQ. 2016. Variable influenza vaccine effectiveness by subtype: a systematic review and meta-analysis of test-negative design studies. Lancet Infect Dis 16: 942-951. doi:10.1016/S1473-3099(16) 00129-8

Biggerstaff M, Cauchemez S, Reed C, Gambhir M, Finelli L. 2014. Estimates of the reproduction number for seasonal, pandemic, and zoonotic influenza: a systematic review of the literature. BMC Infect Dis 14: 480. doi:10.1186/14712334-14-480

Cauchemez S, Valleron AJ, Boëlle PY, Flahault A, Ferguson NM. 2008. Estimating the impact of school closure on influenza transmission from sentinel data. Nature 452: 750-754. doi:10.1038/nature06732

Charu V, Chowell G, Palacio Mejia LS, Echevarría-Zuno S, Borja-Aburto VH, Simonsen L, Miller MA, Viboud C. 2011. Mortality burden of the $\mathrm{A} / \mathrm{H} 1 \mathrm{~N} 1$ pandemic in Mexico: a comparison of deaths and years of life lost to seasonal influenza. Clin Infect Dis 53: 985-993. doi:10 $.1093 / \mathrm{cid} / \mathrm{cir} 644$

Chen YQ, Wohlbold TJ, Zheng NY, Huang M, Huang Y, Neu KE, Lee J, Wan H, Rojas KT, Kirkpatrick E, et al. 2018. Influenza infection in humans induces broadly cross-reactive and protective neuraminidase-reactive antibodies. Cell 173: 417-429.e10. doi:10.1016/j.cell.2018.03.030

Chung JR, Rolfes MA, Flannery B, Prasad P, O’Halloran A, Garg S, Fry AM, Singleton JA, Patel M, Reed C, et al. 2020 Effects of influenza vaccination in the United States during the 2018-2019 influenza season. Clin Infect Dis doi:10 1093/cid/ciz1244

Cooper BS, Pitman RJ, Edmunds WJ, Gay NJ. 2006. Delaying the international spread of pandemic influenza. PLoS Med 3: e212. doi:10.1371/journal.pmed.0030212

Coughlin SS. 2012. Anxiety and depression: linkages with viral diseases. Public Health Rev 34: 7. doi:10.1007/ BF03391675

Cowling BJ, Lau LL, Wu P, Wong HW, Fang VJ, Riley S, Nishiura H. 2010. Entry screening to delay local transmission of 2009 pandemic influenza A (H1N1). BMC Infect Dis 10: 82. doi:10.1186/1471-2334-10-82

Cowling BJ, Ali ST, Ng TWY, Tsang TK, Li JCM, Fong MW, Liao Q, Kwan MY, Lee SL, Chiu SS, et al. 2020. Impact assessment of non-pharmaceutical interventions against coronavirus disease 2019 and influenza in Hong Kong: an observational study. Lancet Public Health 5: e279-e288. doi:10.1016/S2468-2667(20)30090-6

Dave K, Lee PC. 2019. Global geographical and temporal patterns of seasonal influenza and associated climatic factors. Epidemiol Rev 41: 51-68. doi:10.1093/epirev/ mxz008

Davis BM, Aiello AE, Dawid S, Rohani P, Shrestha S, Foxman B. 2012. Influenza and community-acquired pneumonia interactions: the impact of order and time of infection on population patterns. Am J Epidemiol 175: 363-367. doi:10 $.1093 / \mathrm{aje} / \mathrm{kwr} 402$ 
Dowell SF. 2001. Seasonal variation in host susceptibility and cycles of certain infectious diseases. Emerg Infect Dis 7: 369-374. doi:10.3201/eid0703.017301

Erbelding EJ, Post DJ, Stemmy EJ, Roberts PC, Augustine AD, Ferguson S, Paules CI, Graham BS, Fauci AS. 2018. A universal influenza vaccine: the strategic plan for the National Institute of Allergy and Infectious Diseases. J Infect Dis 218: 347-354. doi:10.1093/infdis/jiy103

Farr W. 1847. Tenth annual report of the Registrar General, xii. HMSO, London.

Ferguson NM, Cummings DA, Fraser C, Cajka JC, Cooley PC, Burke DS. 2006. Strategies for mitigating an influenza pandemic. Nature 442: 448-452. doi:10.1038/na ture04795

Fleming DM, Elliot AJ. 2008. Lessons from 40 years' surveillance of influenza in England and Wales. Epidemiol Infect 136: 866-875. doi:10.1017/S0950268807009910

Fong MW, Gao H, Wong JY, Xiao J, Shiu EYC, Ryu S, Cowling BJ. 2020. Nonpharmaceutical measures for pandemic influenza in nonhealthcare settings-social distancing measures. Emerg Infect Dis 26: 976-984. doi:10.3201/ eid2605.190995

Fraser C, Riley S, Anderson RM, Ferguson NM. 2004. Factors that make an infectious disease outbreak controllable. Proc Natl Acad Sci 101: 6146-6151. doi:10.1073/pnas .0307506101

Ginsberg J, Mohebbi MH, Patel RS, Brammer L, Smolinski MS, Brilliant L. 2009. Detecting influenza epidemics using search engine query data. Nature 457: 1012-1014. doi:10.1038/nature07634

Goldstein E, Cobey S, Takahashi S, Miller JC, Lipsitch M. 2011. Predicting the epidemic sizes of influenza A/H1N1, A/H3N2, and B: a statistical method. PLoS Med 8: e1001051. doi:10.1371/journal.pmed.1001051

Hale MJ, Hoskins RS, Baker MG. 2012. Screening for influenza $\mathrm{A}(\mathrm{H} 1 \mathrm{~N} 1) \mathrm{pdm} 09$, Auckland International Airport, New Zealand. Emerg Infect Dis 18: 866-868. doi:10 .3201/eid1805.111080

Hirve S, Newman LP, Paget J, Azziz-Baumgartner E, Fitzner J, Bhat N, Vandemaele K, Zhang W. 2016. Influenza seasonality in the tropics and subtropics-when to vaccinate? PLoS One 11: e0153003. doi:10.1371/journal.pone .0153003

Ip DKM, Lau LLH, Chan KH, Fang VJ, Leung GM, Peiris MJS, Cowling BJ. 2016. The dynamic relationship between clinical symptomatology and viral shedding in naturally acquired seasonal and pandemic influenza virus infections. Clin Infect Dis 62: 431-437.

Iuliano AD, Roguski KM, Chang HH, Muscatello DJ, Palekar R, Tempia S, Cohen C, Gran JM, Schanzer D, Cowling BJ, et al. 2018. Estimates of global seasonal influenzaassociated respiratory mortality: a modelling study. Lancet 391: 1285-1300. doi:10.1016/S0140-6736(17)33293-2

Jackson ML, Nelson JC. 2013. The test-negative design for estimating influenza vaccine effectiveness. Vaccine 31: 2165-2168. doi:10.1016/j.vaccine.2013.02.053

Jackson LA, Jackson ML, Nelson JC, Neuzil KM, Weiss NS 2006. Evidence of bias in estimates of influenza vaccine effectiveness in seniors. Int J Epidemiol 35: 337-344. doi:10.1093/ije/dyi274
Jackson C, Vynnycky E, Hawker J, Olowokure B, Mangtani P. 2013. School closures and influenza: systematic review of epidemiological studies. BMJ Open 3: e002149. doi:10 $.1136 /$ bmjopen-2012-002149

Jewell NP, Lei X, Ghani AC, Donnelly CA, Leung GM, Ho LM, Cowling BJ, Hedley AJ. 2007. Non-parametric estimation of the case fatality ratio with competing risks data: an application to severe acute respiratory syndrome (SARS). Stat Med 26: 1982-1998. doi:10.1002/sim.2691

Kelly H, Cowling BJ. 2013. Case fatality: rate, ratio, or risk? Epidemiology 24: 622-623. doi:10.1097/EDE .0b013e318296c2b6

Kelly H, Cowling BJ. 2015. Influenza: the rational use of oseltamivir. Lancet 385: 1700-1702. doi:10.1016/S01406736(15)60074-5

Khurana S, Hahn M, Coyle EM, King LR, Lin TL, Treanor J, Sant A, Golding H. 2019. Repeat vaccination reduces antibody affinity maturation across different influenza vaccine platforms in humans. Nat Commun 10: 3338. doi:10 .1038/s41467-019-11296-5

Killingley B, Nguyen-Van-Tam J. 2013. Routes of influenza transmission. Influenza Other Respir Viruses 7: 42-51. doi:10.1111/irv.12080

Klepser ME. 2014. Socioeconomic impact of seasonal (epidemic) influenza and the role of over-the-counter medicines. Drugs 74: 1467-1479. doi:10.1007/s40265-0140245-1

Lam EKS, Morris DH, Hurt AC, Barr IG, Russell CA. 2020. The impact of climate and antigenic evolution on seasonal influenza virus epidemics in Australia. Nat Commun 11: 2741. doi:10.1038/s41467-020-16545-6

Lau LL, Nishiura H, Kelly H, Ip DK, Leung GM, Cowling BJ. 2012. Household transmission of 2009 pandemic influenza A (H1N1): a systematic review and meta-analysis. Epidemiology 23: 531-542. doi:10.1097/EDE .0b013e31825588b8

Leung NH, Xu C, Ip DK, Cowling BJ. 2015. Review article: the fraction of influenza virus infections that are asymptomatic: a systematic review and meta-analysis. Epidemiology 26: 862-872. doi:10.1097/EDE.0000000000000340

Leung NHL, Chu DKW, Shiu EYC, Chan KH, McDevitt JJ, Hau BJP, Yen HL, Li Y, Ip DKM, Peiris JSM, et al. 2020. Respiratory virus shedding in exhaled breath and efficacy of face masks. Nat Med 26: 676-680. doi:10.1038/s41591020-0843-2

Li L, Wong JY, Wu P, Bond HS, Lau EHY, Sullivan SG, Cowling BJ. 2018. Heterogeneity in estimates of the impact of influenza on population mortality: a systematic review. Am J Epidemiol 187: 378-388. doi:10.1093/aje/ $\mathrm{kw} 270$

Mak GC, Wong AH, Ho WY, Lim W. 2012. The impact of pandemic influenza A (H1N1) 2009 on the circulation of respiratory viruses 2009-2011. Influenza Other Respir Viruses 6: e6-e10. doi:10.1111/j.1750-2659.2011.00323.x

Marr LC, Tang JW, Van Mullekom J, Lakdawala SS. 2019. Mechanistic insights into the effect of humidity on airborne influenza virus survival, transmission and incidence. J R Soc Interface 16: 20180298. doi:10.1098/rsif .2018 .0298

McLeod MA, Baker M, Wilson N, Kelly H, Kiedrzynski T, Kool JL. 2008. Protective effect of maritime quarantine in South Pacific jurisdictions, 1918-19 influenza pandemic. 
Emerg Infect Dis 14: 468-470. doi:10.3201/eid1403 .070927

Milton DK, Fabian MP, Cowling BJ, Grantham ML, McDevitt JJ. 2013. Influenza virus aerosols in human exhaled breath: particle size, culturability, and effect of surgical masks. PLoS Pathog 9: e1003205. doi:10.1371/journal .ppat.1003205

Monto AS. 1994. Studies of the community and family: acute respiratory illness and infection. Epidemiol Rev 16: 351373. doi:10.1093/oxfordjournals.epirev.a036158

Morris DE, Cleary DW, Clarke SC. 2017. Secondary bacterial infections associated with influenza pandemics. Front Microbiol 8: 1041. doi:10.3389/fmicb.2017.01041

Nicoll A, Ciancio BC, Lopez Chavarrias V, Mølbak K, Pebody R, Pedzinski B, Penttinen P, van der Sande M, Snacken R, Van Kerkhove MD. 2012. Influenza-related deaths-available methods for estimating numbers and detecting patterns for seasonal and pandemic influenza in Europe. Euro Surveill 17: 20162. doi:10.2807/ese.17.18 .20162-en

Nishiura H. 2010. Case fatality ratio of pandemic influenza. Lancet Infect Dis 10: 443-444. doi:10.1016/S1473-3099 (10)70120-1

Noymer A. 2011. The 1918 influenza pandemic hastened the decline of tuberculosis in the United States: an age, period, cohort analysis. Vaccine 29: B38-B41. doi:10.1016/j vaccine.2011.02.053

Pappas C, Aguilar PV, Basler CF, Solorzano A, Zeng H, Perrone LA, Palese P, García-Sastre A, Katz JM, Tumpey TM. 2008. Single gene reassortants identify a critical role for PB1, HA, and NA in the high virulence of the 1918 pandemic influenza virus. Proc Natl Acad Sci 105: 30643069. doi:10.1073/pnas.0711815105

Paules CI, Sullivan SG, Subbarao K, Fauci AS. 2018. Chasing seasonal influenza-the need for a universal influenza vaccine. $N$ Engl J Med 378: 7-9. doi:10.1056/ NEJMp1714916

Payne L, Kühlmann-Berenzon S, Ekdahl K, Giesecke J, Högberg L, Penttinen P. 2005. “Did you have flu last week?” A telephone survey to estimate a point prevalence of influenza in the Swedish population. Euro Surveill 10: 5-6. doi:10.2807/esm.10.12.00585-en

Ryu S, Ali ST, Cowling BJ, Lau EHY. 2020a. Effects of school holidays on seasonal influenza in South Korea, 20142016. J Infect Dis 222: 832-835. doi:10.1093/infdis/ jiaa179

Ryu S, Gao H, Wong JY, Shiu EYC, Xiao J, Fong MW, Cowling BJ. 2020b. Nonpharmaceutical measures for pandemic influenza in nonhealthcare settings-international travel-related measures. Emerg Infect Dis 26: 961-966. doi:10.3201/eid2605.190993

Saad-Roy CM, McDermott AB, Grenfell BT. 2019. Dynamic perspectives on the search for a universal influenza vaccine. J Infect Dis 219: S46-S56. doi:10.1093/infdis/jiz044

Sakaguchi H, Tsunoda M, Wada K, Ohta H, Kawashima M, Yoshino Y, Aizawa Y. 2012. Assessment of border control measures and community containment measures used in Japan during the early stages of pandemic (H1N1) 2009. PLoS One 7: e31289. doi:10.1371/journal.pone.0031289

Saunders-Hastings PR, Krewski D. 2016. Reviewing the history of pandemic influenza: understanding patterns of emergence and transmission. Pathogens 5: 66. doi:10 .3390/pathogens5040066

Sautto GA, Kirchenbaum GA, Ross TM. 2018. Towards a universal influenza vaccine: different approaches for one goal. Virol J 15: 17. doi:10.1186/s12985-017-0918-y

Serfling RE. 1963. Methods for current statistical analysis of excess pneumonia-influenza deaths. Public Health Rep 78: 494-506. doi:10.2307/4591848

Shaman J, Kohn M. 2009. Absolute humidity modulates influenza survival, transmission, and seasonality. Proc Natl Acad Sci 106: 3243-3248. doi:10.1073/pnas .0806852106

Simonsen L, Spreeuwenberg P, Lustig R, Taylor RJ, Fleming DM, Kroneman M, Van Kerkhove MD, Mounts AW, Paget WJ; GLaMOR Collaborating Teams. 2013. Global mortality estimates for the 2009 influenza pandemic from the GLaMOR project: a modeling study. PLoS Med 10: e1001558. doi:10.1371/journal.pmed.1001558

Spreco A, Eriksson O, Dahlström O, Cowling BJ, Timpka T. 2017. Integrated detection and prediction of influenza activity for real-time surveillance: algorithm design. $J$ Med Internet Res 19: e211. doi:10.2196/jmir.7101

Sullivan SG, Feng S, Cowling BJ. 2014. Potential of the testnegative design for measuring influenza vaccine effectiveness: a systematic review. Expert Rev Vaccines 13: 15711591. doi:10.1586/14760584.2014.966695

Talaat M, Afifi S, Dueger E, El-Ashry N, Marfin A, Kandeel A, Mohareb E, El-Sayed N. 2011. Effects of hand hygiene campaigns on incidence of laboratory-confirmed influenza and absenteeism in schoolchildren, Cairo, Egypt. Emerg Infect Dis 17: 619-625. doi:10.3201/eid1704 .101353

Taubenberger JK, Morens DM. 2006. 1918 influenza: the mother of all pandemics. Emerg Infect Dis 12: 15-22. doi:10.3201/eid1209.05-0979

Tellier R, Li Y, Cowling BJ, Tang JW. 2019. Recognition of aerosol transmission of infectious agents: a commentary. BMC Infect Dis 19: 101. doi:10.1186/s12879-019-3707-y

Tokars JI, Olsen SJ, Reed C. 2018. Seasonal incidence of symptomatic influenza in the United States. Clin Infect Dis 66: 1511-1518. doi:10.1093/cid/cix1060

Tsang TK, Lau LLH, Cauchemez S, Cowling BJ. 2016. Household transmission of influenza virus. Trends Microbiol 24: 123-133. doi:10.1016/j.tim.2015.10.012

Van Kerkhove MD, Vandemaele KA, Shinde V, JaramilloGutierrez G, Koukounari A, Donnelly CA, Carlino LO, Owen R, Paterson B, Pelletier L, et al. 2011. Risk factors for severe outcomes following 2009 influenza A (H1N1) infection: a global pooled analysis. PLoS Med 8: e1001053. doi:10.1371/journal.pmed.1001053

Viboud C, Simonsen L, Fuentes R, Flores J, Miller MA, Chowell G. 2016. Global mortality impact of the 1957-1959 influenza pandemic. J Infect Dis 213: 738-745. doi:10 .1093/infdis/jiv534

Warren-Gash C, Smeeth L, Hayward AC. 2009. Influenza as a trigger for acute myocardial infarction or death from cardiovascular disease: a systematic review. Lancet Infect Dis 9: 601-610. doi:10.1016/S1473-3099(09)70233-6

Warren-Gash C, Blackburn R, Whitaker H, McMenamin J, Hayward AC. 2018. Laboratory-confirmed respiratory infections as triggers for acute myocardial infarction and 
S. Ryu and B.J. Cowling

stroke: a self-controlled case series analysis of national linked datasets from Scotland. Eur Respir J 51: 1701794. doi:10.1183/13993003.01794-2017

Wong JY, Kelly H, Ip DK, Wu JT, Leung GM, Cowling BJ. 2013a. Case fatality risk of influenza A (H1N1pdm09): a systematic review. Epidemiology 24: 830-841. doi:10 .1097/EDE.0b013e3182a67448

Wong JY, Wu P, Nishiura H, Goldstein E, Lau EH, Yang L, Chuang SK, Tsang T, Peiris JS, Wu JT, et al. 2013b. Infection fatality risk of the pandemic $\mathrm{A}(\mathrm{H} 1 \mathrm{~N} 1) 2009$ virus in Hong Kong. Am J Epidemiol 177: 834-840. doi:10.1093/ aje/kws314

World Health Organization. 2007. Ethical considerations in developing a public health response to pandemic influenza. World Health Organization, Geneva.

World Health Organization. 2017a. Up to 650,000 people die of respiratory diseases linked to seasonal flu each year. News Release, World Health Organization, Geneva.

World Health Organization. 2017b. Pandemic Influenza Severity Assessment (PISA): a WHO guide to assess the severity of influenza in seasonal epidemics and pandemics. World Health Organization, Geneva.

Wu JT, Riley S, Fraser C, Leung GM. 2006. Reducing the impact of the next influenza pandemic using householdbased public health interventions. PLoS Med 3: e361. doi:10.1371/journal.pmed.0030361

Wu JT, Cowling BJ, Lau EH, Ip DK, Ho LM, Tsang T, Chuang SK, Leung PY, Lo SV, Liu SH, et al. 2010a. School closure and mitigation of pandemic (H1N1) 2009, Hong Kong. Emerg Infect Dis 16: 538-541. doi:10.3201/eid1603 .091216
Wu JT, Ma ES, Lee CK, Chu DK, Ho PL, Shen AL, Ho A, Hung IF, Riley S, Ho LM, et al. 2010b. The infection attack rate and severity of 2009 pandemic H1N1 influenza in Hong Kong. Clin Infect Dis 51: 1184-1191. doi:10.1086/ 656740

Wu JT, Ho A, Ma ES, Lee CK, Chu DK, Ho PL, Hung IF, Ho LM, Lin CK, Tsang T, et al. 2011. Estimating infection attack rates and severity in real time during an influenza pandemic: analysis of serial cross-sectional serologic surveillance data. PLoS Med 8: e1001103. doi:10.1371/jour nal.pmed.1001103

Xiao J, Shiu EYC, Gao H, Wong JY, Fong MW, Ryu S, Cowling BJ. 2020. Nonpharmaceutical measures for pandemic influenza in nonhealthcare settings-personal protective and environmental measures. Emerg Infect Dis 26: 967975. doi:10.3201/eid2605.190994

Yang W, Lipsitch M, Shaman J. 2015. Inference of seasonal and pandemic influenza transmission dynamics. Proc Natl Acad Sci 112: 2723-2728. doi:10.1073/pnas 1415012112

Yu H, Cauchemez S, Donnelly CA, Zhou L, Feng L, Xiang N Zheng J, Ye M, Huai Y, Liao Q, et al. 2012. Transmission dynamics, border entry screening, and school holidays during the 2009 influenza A (H1N1) pandemic, China. Emerg Infect Dis 18: 758-766. doi:10.3201/eid1805 .110356

Zhou Y, Lau EH, Ip DK, Nishiura H, Leung GM, Seto WH, Cowling BJ. 2013. Years of life lost in the first wave of the 2009 influenza A(H1N1) pandemic in Hong Kong. Am J Epidemiol 178: 1313-1318. doi:10.1093/aje/kwt120 


\section{$\&_{\mathrm{CSH}}^{\infty} \&$ Cold Spring Harbor

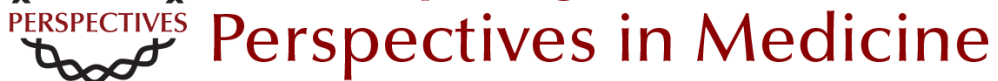

\section{Human Influenza Epidemiology}

Sukhyun Ryu and Benjamin J. Cowling

Cold Spring Harb Perspect Med published online September 28, 2020

Subject Collection Influenza: The Cutting Edge

\section{Emerging HxNy Influenza A Viruses}

William J. Liu, Yan Wu, Yuhai Bi, et al.

Equine Influenza

Thomas M. Chambers

Human Influenza Epidemiology

Sukhyun Ryu and Benjamin J. Cowling

Host Cell Factors That Interact with Influenza

Virus Ribonucleoproteins

Ecco Staller and Wendy S. Barclay

Induction and Evasion of Type-I Interferon

Responses during Influenza A Virus Infection Raquel Muñoz-Moreno, Carles Martínez-Romero and Adolfo García-Sastre

Structure and Function of Influenza Polymerase Joanna M. Wandzik, Tomas Kouba and Stephen Cusack

H7N9 Influenza Virus in China

Chengjun Li and Hualan Chen

H5 Influenza Viruses in Egypt

Rabeh El-Shesheny, Ahmed Kandeil, Ahmed

Mostafa, et al.
Antivirals Targeting the Neuraminidase Larisa Gubareva and Teena Mohan

Accessory Gene Products of Influenza A Virus Rute M. Pinto, Samantha Lycett, Eleanor Gaunt, et al.

Influenza Immunization in the Context of

Preexisting Immunity Susanne L. Linderman, Ali H. Ellebedy, Carl Davis, et al.

Hemagglutinin Structure and Activities Steven J. Gamblin, Sébastien G. Vachieri, Xiaoli Xiong, et al.

Live Attenuated Cold-Adapted Influenza Vaccines Kanta Subbarao

Next-Generation Influenza Vaccines Masaru Kanekiyo and Barney S. Graham

Selective Genome Packaging Mechanisms of Influenza A Viruses Takeshi Noda

Systems Biological Analysis of Immune Response to Influenza Vaccination Mario Cortese, Amy C. Sherman, Nadine G. Rouphael, et al.

For additional articles in this collection, see http://perspectivesinmedicine.cshlp.org/cgi/collection/ 